\title{
Performance Evaluation of Radio-based Train Control System
}

\author{
Hiroshi FUKUOKA \\ Senior Researcher,
}

Kohji IWATA

Assistant Senior Researcher, Signalling Systems Laboratory, Signalling and Telecommunication Technology Division

In general, the railways have kept their systems highly safe and reliable through the ongoing efforts over a period of many years. This has been achieved primarily by continuous improvements, which mean that there is a dearth of experience in the introduction of innovative new systems.

Therefore it is said that comparing a new system that features a completely new architecture with a conventional one may be difficult. With this in mind, we developed a method of evaluating the effect of a new system architecture and took a radio-based train control system as an example. Then we described the result of a case study that evaluated the realtime, safety and reliability properties of the new train control system.

Keywords: performance evaluation, reliability evaluation, real-time evaluation, safety evaluation, radio-based train control system

\section{Introduction}

Railway technology dates back more than a century, and railways have generally maintained highly safe and reliable systems using conventional technologies. These have depended greatly on constant improvements over a period of many years. On the other hand, this means that railways have not experienced the introduction of many completely new system architectures, which railway engineers therefore find difficult to compare with existing technology. For example, a radio-based train control system is said to be more cost-effective and easier to maintain than other train control systems, because it does not depend on the track circuit, which is a fixed blocking system, and, therefore, is able to control trains in a flexible manner.

In addition, various functions can be added to its features so that the radio-based train control system monitors train positions precisely and could then be fully expected to form the basis of a next-generation train control system. However new communication and control system architectures will need to be introduced to realize (1) train control with a radio communication system; (2) real-time control of trains traveling at high-speeds; and (3) network-based control of trains operating over a wide area. There are many cases though that cannot demonstrate any superiority over the conventional system, as a direct comparison with different architectures is inappropriate.

On the other hand, safety levels have recently depended on the new concepts of a safety life cycle and a safety integrity level. In international standard $(\text { IEC62278 })^{1)}$, this is one of the areas in which the reliability, availability, maintainability and safety (RAMS) concept is applied to construct a new high-performance railway system by the integration of various performance parameters for the evaluation of a new train control system.

In a sense, with regard to this trend, it is thought that it is time to prepare a so-called overall railway performance evaluation framework.

Keeping these viewpoints in mind, we are using this paper to discuss the construction of a new method for evaluating train control systems by evaluating the effect of the introduction of a new architecture into the control mechanism, assuming the application of a new train control architecture in a radio-based train control system.

Also, for a case study, we describe the result of a performance evaluation of a radio-based train control system, in which a radio-based train control system is shown to be superior from the safety viewpoint.

\section{Overview of train control system evaluation methods}

Simulators have been developed to evaluate the performance of train control systems. These evaluate the service methodology and control system of trains by detailed simulation of train operations and movements.

For this paper, which takes the evaluation of a new radio-based train control system as its starting point, we developed a simulator that enables the overall system performance to be assessed, with evaluations carried out of the control/operation and communication methods, real-time property, communication network/ protocol and communication system traffic. Figure1 provides an overview of the train control evaluation system.

As described in Fig. 1, the train control evaluation simulator (Section 3, SIMulator for platform of TRAin control System, SIMTRAS) simulates train operations and movements and evaluates various overall performance parameters. The safety logic analysis system (Section 4, RiskNet) evaluates safety logic performance, with a general-purpose communication simulator (Section 5, Network Simulator 2, ns2) ${ }^{2)}$ used to evaluate the communication system. 


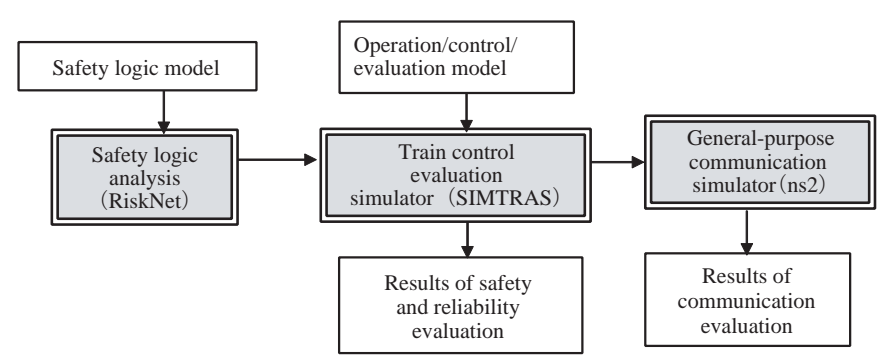

Fig. 1 Overview of train control evaluation system

\section{Train control evaluation simulator}

\subsection{Special features of train control evaluation simulator}

If it is intended to evaluate the performance of an entirely new radio-based train control system, it is necessary to evaluate the real-time properties of a control/ operation method, a communication system and a communication network protocol. Therefore we developed a system that can represent not only the relationships between the times of events that progress in parallel but also relative geometric relations (SIMTRAS system).

\subsection{Simulation control}

This simulator is of an event-driven type. The simulator's main purpose is the simulation of train operations/movements, which has two schedulers (one for time and another for position) that are implemented using separate databases for time and position.

The time scheduler, which has the time database for time events (train departures, radio communication times, etc.), selects and performs the next events. This scheduler ensures that only one event is executed at any one time. If more than two different events are to be executed simultaneously, an event scheduled early (or started early if the events were scheduled at the same time) has a priority. On the other hand, the position scheduler has train and station positions in the position database. This selects and performs the next event, according to the progress of events (in most cases dictated by train movements).

Continuous time events like train movements are approximated by discrete time events and then approximated and represented by a discrete time- and eventdriven type simulator.

\subsection{Evaluation objectives}

Basically, in this section we evaluate the real-time property architectures, the safety and reliability of the train control mechanisms as well as their index-based management methodology. That is to say, we focus on the train operation, signalling system (blocking systems of the moving block type, etc.), communication network configuration (including communication traffic speed), communication protocol, control methods for real-time operations and so on.
Evaluation measurements, mainly related to train operation for railway traffic, are as follows:

(1) Hazard evaluation

(hazard: a physical situation that is potentially harmful to humans or property, likely to cause accidents)

For the safety measurements, we calculate the risk of the following hazards:

(a) Collision between trains, derailment, collision hazard (other than between trains)

(b) Platform-train interface

(c) Railroad level crossing

(d) Natural disaster and others

(2) Reliability

We focus on delayed train departures from stations caused by system shutdowns following safety system activation or railway system failure.

(3) Real-time property

Assuming a real-time control system in which transmitted messages have valid periods (for example, a few seconds after transmission), we thought about a probability of exceedance that execution period would overrun the deadline due to the message validity. We also considered overall train delays in relation to unscheduled train stops due to the real-time control system mentioned above.

\section{Analysis of safety logic}

\subsection{Safety management with safety integrity level ${ }^{3)}$}

Systems that are required to have high safety margins often incorporate a safety management methodology based on the new safety life cycle and safety integrity level concepts.

The safety life cycle methodology is a definition of a development process of a safety-related system, which defines the concept design, design, manufacturing, operation as well as maintenance and improvements phases as a safety life cycle, and includes a safety management activity definition for each phase.

The safety integrity level is defined by the risk, expressed by the occurrence rate and consequence of a hazard. Technological requirements are defined in conjunction with the safety integrity level, selected in accordance with the required safety level.

The safety management activities are as follows. At first, as a safety analysis, the basic system specification is defined and hazards are enumerated. Then a hazard analysis is performed to specify the components and the configuration of the hazard. Also, a risk analysis is undertaken to evaluate the safety level of the system.

Next, safety requirement specifications will be defined, based on the results of the risk analysis. In the safety requirements specification, the target safety integrity level and functions necessary to achieve the required safety level must be defined.

In the following sections, safety analysis methods for the train control evaluation system are discussed, based on the concepts of the above-mentioned safety management methods. 


\subsection{Safety analysis procedures}

The safety analysis methods used in the safety life cycle activities mentioned in the previous section are as follows:

(1) Enumeration of hazards

To clarify the system construction and take all of the hazards into consideration, the system configuration is defined and potential hazards enumerated.

(2) Hazard identification

Hazards to be analyzed are identified by quantitative evaluation and/or qualitative analysis.

(3) Definition of safety logic

Safety measurements are clearly defined for each specified hazard.

\subsection{Network-type safety evaluation model}

Generally, for the safety analysis procedures mentioned above, the safety system configuration is mainly treated as a logic model. In the field of safety evaluation, event tree (ET) and fault tree (FT) analysis models are often used for such logic models, but in cases where tree-shaped logic representation is used, the problem arises that the relations between the states may not be so explicitly defined.

Therefore, to overcome this problem, RTRI developed the typed state influence diagram (TSID, Fig. 2) ${ }^{4)}$ as a new network-type reliability model, which can dynamically define types of relations between states, if required, and explicitly represent these relations.

This network-type reliability model was introduced to provide a description of a safety evaluation model. In addition, this model explicitly and directly defines relations (operators) between each system state. A general functional relation can be defined as an operator of this model. But, to take the advantage of high-speed calculations, here we introduced relations between states according to normal logic models ("and", "or", "branch" and "transition").

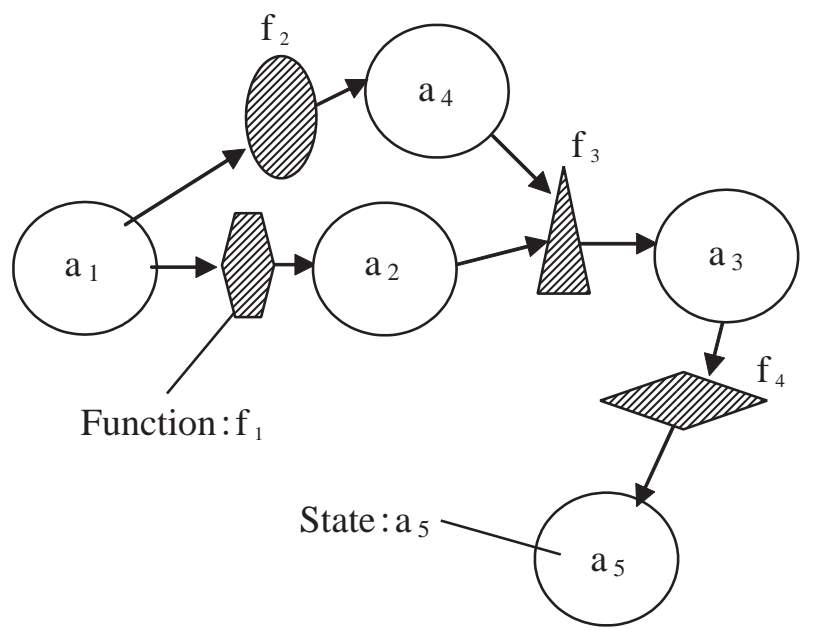

Fig. 2 Typed State Influence Diagram (TSID)

\subsection{Safety logic analysis system}

To evaluate safety, we used the logic evaluation system to analyze the safety logic described in the form mentioned in the previous section. For this we developed a system, which runs on a personal computer, to process the above-mentioned logic analysis (RiskNet system).

Algorithms for this analysis system are, firstly, a safety evaluation model expressed as a TSID model, which is converted to a Boolean expression diagram (BED) model 5) using "UP" algorithm, the BED model then being converted to a binary decision diagram (BDD) logic model to calculate safety measurements.

In addition, the logic model has non-coherency because a "branch" operator is introduced as a function of the relation between states, as mentioned above. The analysis algorithm using the BDD model provides for this type of logic model. In addition, this algorithm has a special feature to give the exact solution; in contrast, an ordinary algorithm using cut sets gives only an approximation.

For the safety evaluation, firstly, safety logic data will be analyzed by a safety logic analysis system (RiskNet), as shown in Fig. 3. The SIMTRAS train control evaluation simulator accepts the result of this analysis in the form of a BDD model and analyses the overall safety of the train control/operation system.

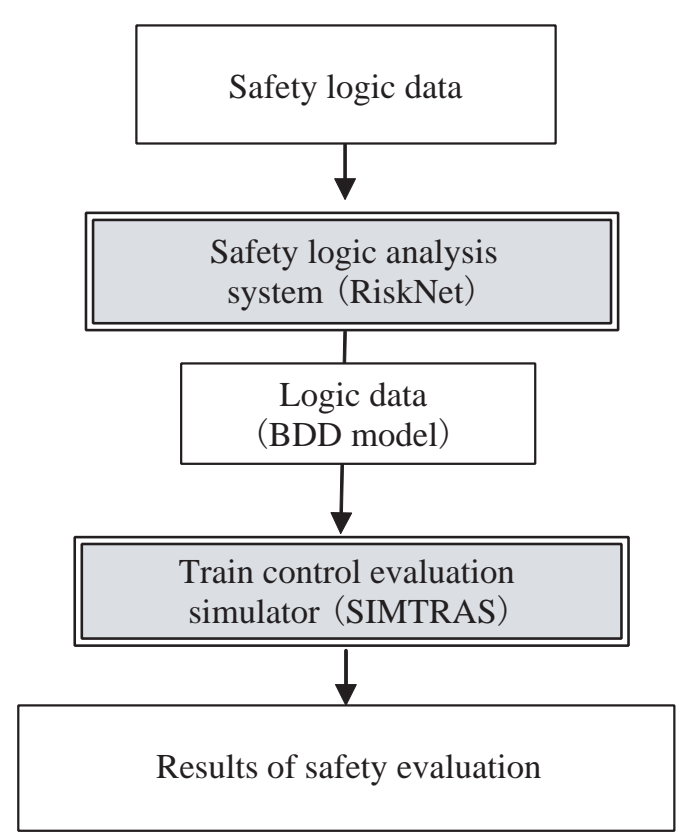

Fig. 3 Interface between safety logic analysis system and train control evaluation simulator

\section{Real-time property evaluation of communication system}

\subsection{Communication simulator: ns2}

A communication model was evaluated using the Network Simulator 2 (ns2) system ${ }^{6}$, a general-purpose communication simulator and discrete event simulator developed for communication network study, which was derived from the REAL network simulator in 1989 and supported as a U.S. Defense Advanced Research Projects 
Agency (DARPA) project in 1995. In recent years, it has also been supported by another DARPA project, and one conducted by the U.S. National Science Foundation (NSF).

Over the last few years, significant progress has been made with the system, which supports a transmission control protocol (TCP) simulation of a wired/radio communication (local or satellite) network, routing and a multicast protocol.

\subsection{Cooperation with ns2}

Simply put, the ns2 system simulates communications based on the train movement log and the transmission (reception) communication log derived from the train operation simulator. The communication log, which is an output of the $\mathrm{ns} 2$, is then analyzed to evaluate the communication system (Fig. 4).

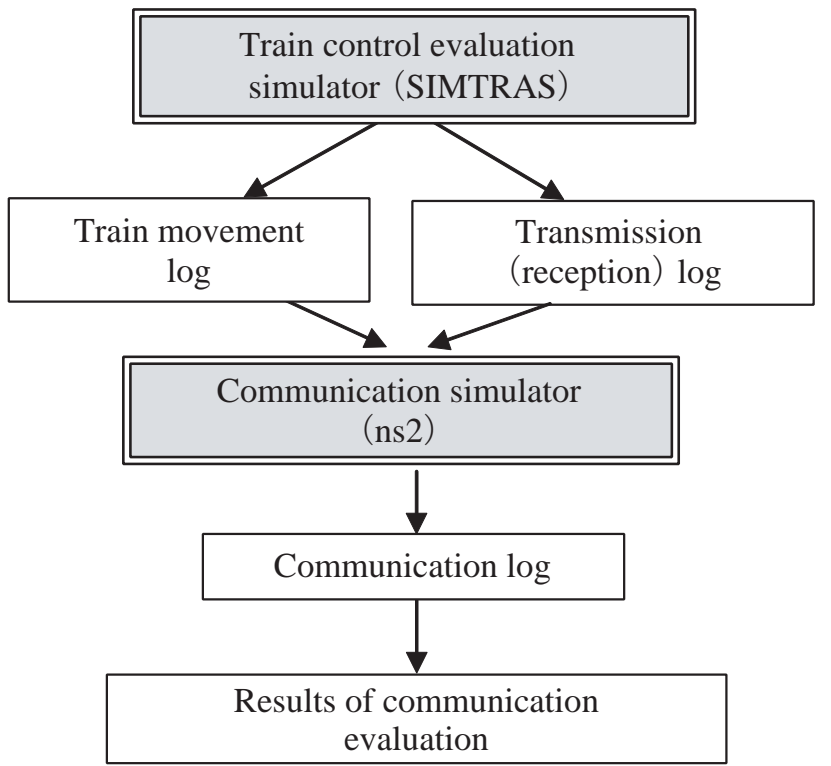

Fig. 4 Interface between train control evaluation and communication simulators

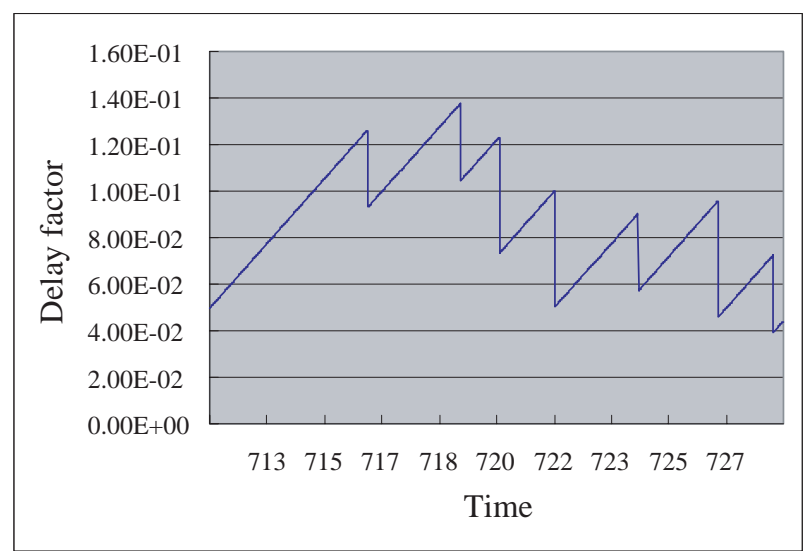

(1) Ordinary speed control

\section{Case study}

\subsection{Assumptions}

For a case study, supposing a commuter line on a loop-type network with high traffic density during the commuter rush hour, we defined the applied system configuration as follows:

(1) Train control system:

A radio-based control system

(2) Train operation system:

Same as commonly found on existing commuter trains

(3) Position monitoring system:

Based on detection of running gear rotation, compensated by transponders

(4) Radio network:

Time division radio network

(5) Wired network:

Same as existing LAN network for signalling systems

(6) Ground device:

Same as existing device, such as ATC ground device, etc.

\subsection{Safety and reliability evaluation}

For the system mentioned in the previous section, we supposed the following train operations. The service begins as normal from the first train, but it is assumed that something delays a train at a station by about 20 minutes at around 6:50 a.m. (which is at the start of the commuter rush hour and also about two hours after the first train). We developed a reliability measurement for a train delay and a safety measurement for a train collision, using the following two control methods:

(1) Ordinary speed control

(2) Smooth control of following trains (taking into consideration that the preceding train is stopped at a station, a following train run at a slower speed)

Evaluated reliability measurements (delay factor: the value proportional to the overall delay for station departures) and evaluated safety measurements (integrity

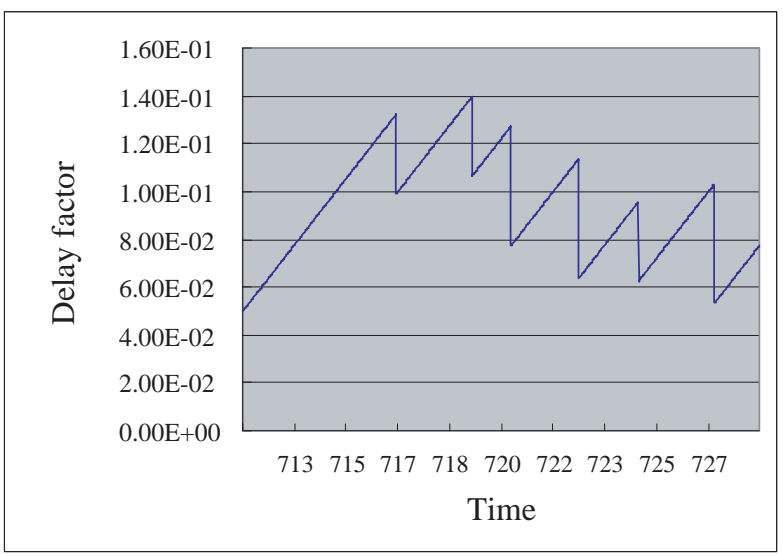

(2) Smooth control of following trains

Fig. 5 Evaluated reliability measurements

(Time : "727" means "7:27 a.m." for example) 


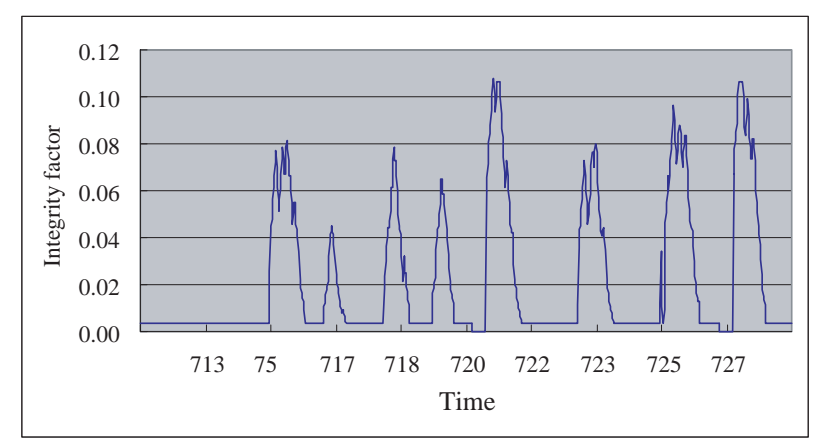

(1) Ordinary speed control

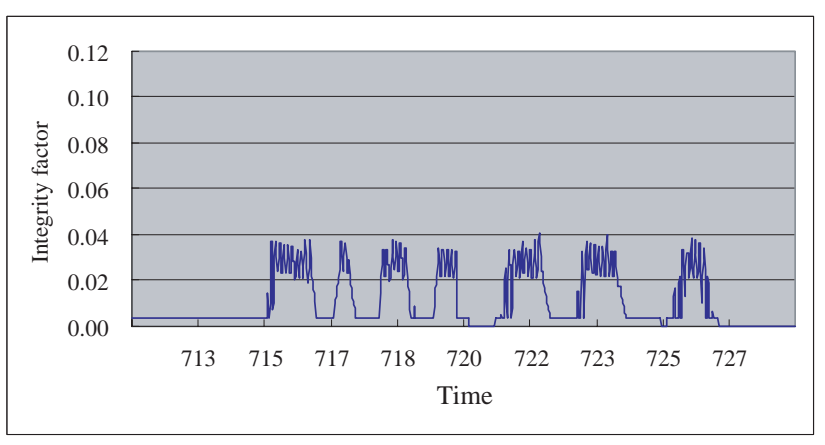

(2) Smooth control of following trains

Fig. 6 Evaluated safety measurements

(Time : "727" means "7:27 a.m." for example)

factor: a value proportional to overall risk according to a collision between trains) are shown in Fig. 5 and Fig. 6 , respectively. For the reliability measurements, in both of cases (1) and (2), a delay occurs after the initial incident and converges afterwards, but the difference between the two cases is very small. On the other hand, the safety measurements for case (2) seem to be much lower than those of case (1).

These results mean that, for the blocking control of an ordinary track circuit (a fixed block system), it may happen that the trains run with a minimum safety margin (a following train approaches a preceding train so closely, across the blocking border), because the following train does not know the precise position of the preceding train in that blocking section. On the other hand, in the case of the blocking control on the radio-based system (moving block), the following train always knows the precise position of the preceding train, and then the maximum safety margin can be taken to its fullest extent according to the transportation requirements. In other words, the radio-based train control system can often control trains effectively from the safety viewpoint, because its controls are based on exact information on the movement of the preceding trains.

However, it should be pointed out that the results mentioned above refer to the hazards of trains running in platoons. The analysis is based on the relation between the safety margins and the approach of the trains, where margins are beyond the safety system limits provided to prevent a train collision.

\subsection{Evaluation of real-time property of a communication system}

Under the same assumptions as Section 6.1, relatively light train traffic on train operations from the first train until about 6:30 a.m. is supposed. We simulate the communication traffic between trains and one ground device with a popular radio LAN system, and then evaluated the distribution function according to communication delays for packets using an exponential distribution approximation (Fig. 7).

This Figure shows that in this communication system, using a radio LAN system, no real-time system is introduced, but it is estimated that the maximum delay

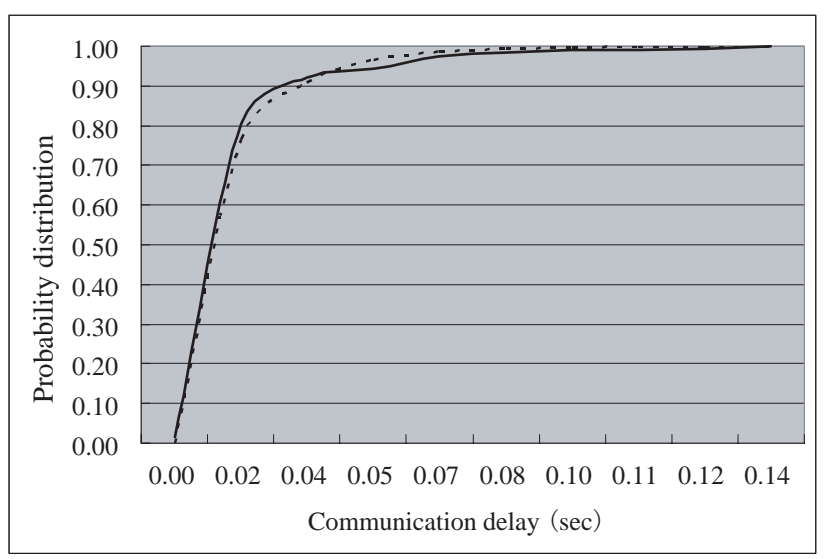

Fig. 7 Results for real-time property evaluation of radio communication system

(Probability distribution of communication delays: Solid line represents results of simulation, dotted line does approximated by exponential distribution)

would be a few seconds, when real-time communication is allowed for.

\section{Conclusions}

An overall performance evaluation framework is going to be fixed for railway systems by the appearance of RAMS indexes as an international standard. In this paper, we built performance evaluation parameters within this framework and described the technique to evaluate it for a train control system featuring new architecture such as a radio-based train control system. Firstly for the safety logic analysis, we discussed the RiskNet safety logic evaluation system, which is based on TSID, the network-type reliability model used to represent complicated dependences.

For the evaluation of various performance indexes of a train control system (especially for the new concept of a radio-based train control system), we developed a SIMTRAS simulation system to evaluate real-time, safety and reliability properties.

Some case studies were carried out to demonstrate that these frameworks are effective for the development 
of the entirely new radio-based train control system, which was shown to be superior from the safety viewpoint.

\section{References}

1) IEC62278: "Railway Applications - The Specification and Demonstration of Reliability, Availability, Maintainability and Safety (RAMS)," September 2002, (CENELEC EN50126)

2) The University of Southern California's Information Sciences Institute (ISI), "The Network Simulator ns2," http://www.isi.edu/nsnam/ns/
3) Iwata, K., et al. "Prospective Safety Analysis of CARAT," RTRI Report, Vol.13, No.8, pp.39-44, 1999 (in Japanese)

4) Fukuoka, H.: "Reliability Evaluation Method for the Railway System: A Model for Complicated Dependency," Quarterly Report of RTRI, Vol.43, No.4, 2002

5) Andersen, H. R. and Hulgaard, H.: "Boolean Expression Diagrams," LICS '97 (12th Symposium on Logic in Computer Science), Warsaw, Poland, 1997

6) Fukuoka, H.: "Uncertainty Analysis in Railway Safety Assessment," Quarterly Report of RTRI, Vol.40, No.1, 1999 